\title{
UMA ANÁLISE PRELIMINAR DO DOCUMENTO HISTÓRICO REGULA DE ABACO COMPUTI DE AUTORIA DO MATEMÁTICO GERBERT DE AURILAC (976 d.C)
}

\author{
A PRELIMINARY ANALYSIS OF THE ABACO COMPUTI REGULA \\ HISTORICAL DOCUMENT OF THE MATHEMATICAL AUTHORITY OF \\ GERBERT DE AURILAC (976 AD)
}

\author{
Suziê Maria de Albuquerque ${ }^{1}$ \\ Instituto Federal de Educação, Ciência e Tecnologia do Ceará \\ Ana Carolina Costa Pereira ${ }^{2}$ \\ Universidade Estadual do Ceará
}

\begin{abstract}
Resumo
O presente artigo aborda reflexões que partiram da pesquisa de mestrado que está sendo desenvolvida no Instituto Federal de Educação, Ciência e Tecnologia do Ceará, com o intuito de estudar as potencialidades didáticas da fonte histórica Abaco Computi, escrita pelo matemático Gerbert de Aurilac no século X. Neste trabalho buscou-se realizar uma descrição histórica da fonte em questão, conhecendo fatores que influenciaram na forma de sua escrita e divulgação científica. Para tanto, além do Abaco Computi (976), contido na obra Ouvres de Gerbert de Olleris (1867), tivemos acesso a trabalho de historiadores que como Chasles (1843a, 1843b), Ferreira (2008), Vasconcelos (1910), Havet (1889) e Loupot (1869) que estudaram o autor, seu contexto histórico e métodos de conhecimento científico. A metodologia desta pesquisa foi a documental, fundamentada no estudo de uma fonte histórica, ou seja, um documento histórico. A partir da pesquisa realizada pudemos identificar que a pesar do momento histórico da idade média, Gerbert produziu uma matemática própria para seu tempo, a exemplo dos registros no Abaco Computi que foram feitos sob encomenda, para facilitar a compreensão do monge Constantino sobre o uso da Tábua do Ábaco com o mecanismo das fichas utilizando dígitos para representar as quantidades, adotando o sistema decimal e posicional de numeração, enfatizando a participação dessa fonte no ensino de aritmética da época em que foi escrita, indicando o caráter instrucional do documento estudado de maneira a trazer à tona a busca por, a partir do conhecimento da estrutura deste trabalho, se pensar em adaptações para o ensino de matemática nos dias atuais.
\end{abstract}

Palavras-chave: História da Matemática; Fonte Histórica; Abaco Computi.

\section{Abstract}

This article deals with reflections from the master 's research that is being developed at the Federal Institute of Education, Science and Technology of Ceará, in order to study the didactic potentialities of the Abaco Computi, written by the mathematician Gerbert de Aurilac in the 10th century In this work we have tried to make a historical

\footnotetext{
${ }^{1}$ suziealbuquerque@hotmail.com

2 carolina.pereira@uece.br
} 
description of the source in question, knowing factors that influenced the form of its writing and scientific dissemination. In addition to Abaco Computi (976), contained in the Ouvres work of Gerbert de Olleris (1867), we had access to the work of historians such as Chasles (1843a, 1843b), Ferreira (2008), Vasconcelos (1910), Havet and Loupot (1869) who studied the author, his historical context and methods of scientific knowledge. The methodology of this research was the documentary, based on the study of a historical source, that is, a historical document. From the research we have been able to identify that despite the historical moment of the Middle Ages, Gerbert produced a mathematics of his own, like the records in the Abaco Computi that were made to order, to facilitate the understanding of the monk Constantine on the use of Abacus Table with the mechanism of the fiches using digits to represent the quantities, adopting the decimal and positional system of numbering, emphasizing the participation of this source in the teaching of arithmetic of the time in which it was written, indicating the instructional character of the document studied in a way to bring to the fore the search for, from the knowledge of the structure of this work, to think about adaptations for the teaching of mathematics in the present day.

Keywords: History of Mathematics; Historical Source; Abaco Computi.

\section{Introdução}

A história da matemática como área de conhecimentos revela potencialidades didáticas e pedagógicas, Baroni, Teixeira e Nobre (2004) apresentam pontos relevantes na contribuição de melhorias no ensino de matemática, dentre elas podemos destacar de forma mais abrangente a compreensão do desenvolvimento das ciências e da matemática com visão não linear, contrariando a lógica tradicional do desenvolvimento histórico das ciências, até então disseminada.

No enfoque escolar, os professores têm dificuldades quanto ao acesso a fontes em História da Matemática, considerando, por exemplo, que os livros didáticos são por vezes o principal material que subsidiam o planejamento das aulas. No entanto, esses materiais apresentam a História da Matemática de acordo com uma visão da História Tradicional, dificultando a abordagem Historiográfica Atualizada, tendo em vista que o estudo historiográfico "fica enriquecido quando mobilizamos, de forma articulada, o maior número e tipos de fontes possíveis" (GARNICA; SOUZA, 2012, p. 42).

Pereira e Pereira (2015) abordam a questão da pesquisa em História da Matemática em seu artigo sobre fontes históricas:

A função do pesquisador em História da Matemática é então descobrir as origens e em seguida a evolução de ideias, noções e métodos científicos, "desdogmatizando" a formação Matemática, sendo muitas vezes imparcial 
com situações que possam oferecer dúvidas (PEREIRA; PEREIRA, 2015, p. $70)$.

A pesquisa em História da Matemática amplia as possibilidades de existência de materiais didáticos voltados para o ensino, na medida em que o pesquisador busca nas origens, fontes primárias, subsídios para que os educadores matemáticos possam elaborar atividades que explorem esses documentos históricos.

Esteve (2011) traz a ideia de que a História da Matemática se apresenta como Recurso Didático para a compreensão da matemática, ressaltando que um dos campos de aplicação da história da matemática em sala de aula diz respeito ao uso de textos históricos para melhorar a compreensão dos conceitos matemáticos, potencializando as atividades didáticas, colaborando com as ideias de Pereira e Pereira (2015).

O diálogo entre História e Educação Matemática impele ao rompimento do paradigma de que a matemática é uma área isolada em si mesma, puramente exata, favorecendo a ideia da humanização da História da Matemática, do estudo de como as comunidades produziam e compartilhavam conhecimentos no passado para poder avaliar os acontecimentos do presente, reorientando as práticas educativas em Ensino de Matemática.

Além disso, pode colaborar para que os professores ampliem sua formação docente, produzindo subsídios metodológicos, contribuindo para que estudantes compreendam que a matemática é mais do que um conjunto de regras, se trata de uma produção histórica e social ao longo do curso histórico de evolução da humanidade.

Diante do exposto, este artigo tem por objetivo descrever a fonte primária Ábaco Computi, escrita pelo matemático Gerbert de Aurilac, por volta do ano 976 d.C, na França, influenciando as formas de cálculos aritméticos realizadas no período histórico de sua elaboração, conduzindo ao conhecimento e organização da fonte histórica para que esta possa ser utilizadas pelos professores na implementação de atividades escolares.

\section{Abordagem Metodológica}

O presente estudo foi desenvolvido com a metodologia da pesquisa documental, pois "a característica da pesquisa documental é que a fonte de coleta de dados está restrita a documentos, escritos ou não, constituindo o que se denomina de fontes 
primárias." (LAKATOS; MARCONI 2003, p. 174). Neste caso, trata-se de uma análise preliminar do documento original Abaco Computi, uma fonte primária do século X.

A definição metodológica direcionou ao aprofundamento sobre o conceito de fonte histórica como meio de contextualizar o tipo de material que está sendo investigado, pois este requer manuseios e análises específicas de acordo com suas características, situando a época histórica de elaboração dos textos, tendo em vista que:

\footnotetext{
Fontes históricas são os vestígios do passado, deixados por sociedades e agentes, que nos permitem desvendar seus hábitos, seus costumes, suas produções culturais e, principalmente, suas formas de organização. Dentre os tais vestígios, documentos, manuscritos, livros e restos arqueológicos são apenas algumas das fontes históricas a partir das quais historiadores e pesquisadores da história das ciências, inclusive da história da matemática, trabalham constantemente (GARNICA; SOUZA, 2012, p. 27).
}

No contexto da pesquisa, partimos do documento histórico na perspectiva não linear, ou seja, buscando identificar os processos de construção do conhecimento produzido e difundido por meio dos vestígios historiográficos com os quais o manuscrito Abaco Computi se encarregou de preservar ao longo de mais de um milênio.

Garnica e Souza (2012) estabelecem ainda uma reflexão sobre a diferença entre fontes e documentos históricos. Segundo estes autores, uma fonte passa a ser um documento no momento em que esta se torna objeto de estudo de uma pesquisa. Sendo assim, podemos dizer que a partir desse estudo preliminar sobre o Abaco Computi este se constitui um documento histórico.

Esta investigação teve início com a necessidade de se compreender os métodos de cálculos aritméticos a partir do Ábaco utilizado por Gerbert de Aurilac, iniciando a busca pelo conteúdo do manuscrito Abaco Computi de autoria deste matemático no qual havia detalhado os mecanismos de manuseio do instrumento que eram praticados na época histórica em questão.

Gerbert era francês, viveu na Europa medieval do século $\mathrm{X}$ e teve atuação científica significativa nas áreas do Quadrivium (aritmética, geometria, música e cosmologia). Sendo assim, sua produção foi explorada por historiadores franceses aos quais realizamos consultas a partir de publicações registradas na Biblioteca Virtual Gallica, local onde tivemos acesso ao documento original Regula Do Abaco Computi que faz parte de uma coletânea de produções de Gerbert organizada por Olleris (1867), 
no qual contém desde as cartas eclesiais aos documentos científicos deste matemático notável.

O texto histórico está escrito em latim, no entanto, em uma versão atualizada em relação ao latim medieval, estamos em processo de tradução para o português, etapa que foi dada prosseguimento com o intuito de viabilizar a construção deste artigo. A tradução do documento se constitui em ponto relevante para este trabalho e estudos posteriores.

A partir do contexto histórico no qual o documento original foi elaborado, se tornou possível conhecer de forma preliminar a estrutura do escrito histórico, apontando indícios da matemática que era produzida e ensinada na época de sua elaboração para podermos visualizar meios de apropriação desses achados de maneira que essas construções sejam incorporadas no Ensino de Matemática atual.

\section{O contexto histórico de elaboração e elementos da fonte primária Abaco Computi}

Com o intuito de vincular informações sobre os processos de desenvolvimento da aritmética vamos trazer à tona a história do religioso e matemático Gerbert de Aurilac que haveria de se tornar Silvester II, papa da Igreja Católica (999 d.C a 1003 d.C). Antes de se tornar Papa, Gerbert viveu em Aurilac, cidade que pertencia à França e era na época um centro de conhecimentos matemáticos com estudiosos da aritmética, geometria, astronomia e música, tendo contato com culturas mulçumanas, contribuindo para a socialização de saberes e construção de novos conhecimentos.

No movimento de consolidação e elaboração de conhecimentos, Gelbert escreveu obras matemáticas como: "De Abaco Computi, De numerum divivione, Geometria, Carta a Adebold o cálculo da área de triângulos, a constantin sobre a esfera, dentre outras cartas" (FERREIRA, 2008, p. 45). Ou seja, houve considerável número de produções matemáticas por parte de Gerbert e seus contemporâneos que podem nos ajudar a compreender melhor os processos de construção do conhecimento matemático a partir dessas obras que se tornaram fontes históricas e constam de registros que expressam a forma de pensar matematicamente de um povo, em um tempo e espaço.

De acordo com Ferreira (2008), Gerbert juntamente com seus seguidores elaboraram métodos de multiplicação e divisão adotando o sistema posicional do ábaco. 
No entanto, pelo fato de utilizar uma simbologia própria para cada quantidade, os algarismos hindu-arábicos e representando-os no ábaco foi pouco compreendido na época por introduzir entes abstratos ao sistema concreto e manipulável do ábaco.

Neste trabalho iremos enfatizar que o Tratado Abaco Computi foi escrito pelo matemático Gerbert de Aurilac por volta do ano 976 d.C. Os manuscritos de Gerbert foram transcritos, digitalizados e divulgados em biblioteca virtual, facilitando o acesso à fonte primária e principal que subsidiará reflexões a cerca do objeto de estudo da pesquisa proposta neste projeto. A publicação da fonte original que iremos estudar é do historiador francês Olleris (1867).

Vasconcelos (1910) colabora de maneira específica com significado de Abaco Comuputi, trazendo sua importância para a disseminação dos algarismos hindu arábicos na Europa Medieval e a colaboração de Gerbert no aperfeiçoamento da utilização dos caracteres utilizados para a representação numérica.

Segundo Loupot (1869), Gerbert era professor dos nobres, elaborava instrumentos para aproximar os conhecimentos de astronomia e matemática das pessoas. A maneira encontrada por Gerbert de tornar a aritmética de fácil compreensão foi por meio da aplicação dos métodos de cálculo no ábaco. As variantes de manuseio deste instrumento são especificadas pelo matemático em seu tratado que é nosso presente objeto de estudo.

Os escritos do matemático Gerbert de Aurilac passaram pelos cuidados de historiadores que se incumbiram de sua organização para torna-los acessíveis. Havet (1889) aborda a disposição dos textos de Gerbert que Olleris (1867) coletou de seus manuscritos:

Em 1867, M. Olleris publicou as "Obras completas de Gerbert", fruto de longos anos de estudos. As variantes que ele extraiu de um manuscrito da Biblioteca da Universidade de Leiden ( $\mathrm{n}^{\circ} 54$ da coleção Vossius) permitiram que ele desse uma edição mais correta da correspondência de Gerbert. Mas M. Olleris teve a infeliz ideia de não respeitar a ordem em que essas cartas foram organizadas nos manuscritos. Sua classificação é feita sem nenhum fundamento realmente científico (HAVET, 1889, p. 237, tradução nossa). ${ }^{3}$

\footnotetext{
${ }^{3}$ En 1867, M. Olleris publia les «OEuvres complètes de Gerbert », fruit de longues années d'études. Les variantes qu'il tira d'un manuscrit de la Bibliothèque de l'Université de Leyde ( ${ }^{\circ} 54$ du fonds Vossius) lui permirent de donner une édition plus correcte de la correspondance de Gerbert. Mais M. Olleris eut l'idée malheureuse de ne pas respecter l'ordre où ces lettres étaient rangées clans les manuscrits. As classification est faite sans fondement vraiment scientifique (HAVET, 1889, p. 237).
} 
A partir da produção de Havet (1989) tornou-se possível visualizarmos que além de Olleris outros historiados transcreveram os textos de Gerbert, porém Olleris (1867) foi quem conseguiu reunir o maior número de escritos e de forma mais organizada.

Entretanto, a pensar das críticas quanto aos critérios cronológicos para a organização dos seus trabalhos e fatos históricos contidos neles (comunicações religiosas e material científico). Olleris (1867) tornou acessível a transcrição do tratado Abaco Computi que deixou de ser uma obra isolada para fazer parte de uma coletânea de textos. É relevante se ter noção da obra como um todo para darmos conta do contexto histórico de elaboração do documento pesquisado, fatores sociais e culturais que de alguma forma influenciaram o autor no momento da escrita historiográfica.

No caso, estamos estudando um tratado que é parte da Obra Maior "Oeuvers de Gerbert Pape sous le nom de Sylvestre II: collationnées sur les manuscrits”. Vejamos as seguintes informações que se apresentam na capa da coletânea de escritos de Gerbert por Olleris (1867) que foi digitalizada e se encontra na biblioteca francesa Gallica, contendo sua bibliografia, notas críticas e históricas.

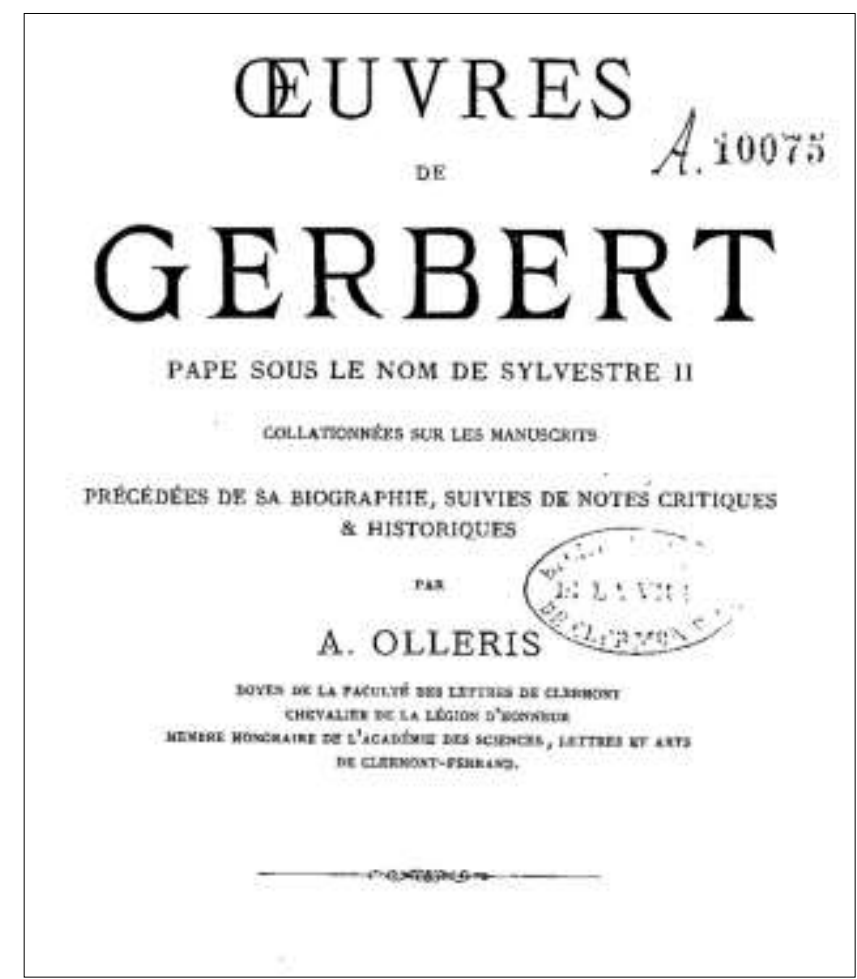

Figura 01 - Capa da coletânea de escritos de Gerbert que contém o Ábaco Computi Fonte: Olleris, capa (1867) 
A descrição do documento histórico em questão está permeada de elementos culturais na construção do conhecimento matemático que impele a busca por compreensão a partir de outros escritos do autor, a fim de situar o texto no contexto histórico e apresentar a dimensão da forma de pensamento do autor, considerando que seus textos representam uma forma de comunicação ao longo dos tempos.

[...] Gerbert conseguiu formar uma importante biblioteca com as cópias de grande número de obras clássicas latinas, e ele próprio compôs muitas obras científicas em que se compreendem: um tratado sobre ábaco - Regula de abaco computi - com o aperfeiçoamento resultante do emprego de caracteres diferentes ou ápices, para cada um dos números de 1 a 9 , que permitiam apresentar os números da mesma maneira que com as cifras Gobar (mas sem o símbolo para zero) que os árabes adotaram, derivando-as das cifras Devaganari, da Índia (VASCONCELOS, 1910, p. 622).

Vasconcelos (1910) destaca o caráter de aprimoramento de técnicas aritméticas proposto por Gerbert a partir de suas produções que tornam públicas por meio de seus manuscritos destinados, segundo Chasles (1843b), ao monge Constantino.

A partir dos relatos de Loupot (1869) é evidenciado o caráter instrucional e voltado para o ensino, devido à função de professor a que era atribuída a Gerbert, mesmo cumprindo suas funções eclesiásticas. Assim, a motivação de elaboração do documento Abaco Computi emergiu da necessidade de facilitar os métodos de ensino e melhor instruir seus alunos na iniciação à aritmética.

O Abaco computi está em processo de tradução, partindo do idioma original que é o latim do século X. Portanto, para fins de conhecimento mais detalhado de seus elementos esse estudo tomou como base relatos de historiadores que tiveram acesso ao documento. No entanto, como dado de pesquisa, a única cópia que tivemos acesso até o momento foi a de Olleris (1867).

Loupot (1869) e Brown (2010) relatam a estrutura instrumento do ábaco que é explorado no documento primário. Trata-se de uma tábua com vinte e sete colunas verticais agrupadas de três em três, onde os nove símbolos que Gerbert havia se apropriados dos árabes eram distribuídos, de acordo com o valor das quantidades. $\mathrm{O}$ símbolo inserido imediatamente a esquerda tinha maior valor posicional. Como os árabes faziam, as inscrições eram acrescentadas da direita para a esquerda. Este indício histórico reforça a origem oriental do nosso sistema de numeração. Iniciando com as 
unidades, dezenas, centenas, unidade de milhar, centena de milhar e assim sucessivamente.

Desta maneira o livro do ábaco continha regras de cálculo que poderiam ser aplicadas para facilitar o uso do ábaco:

[...] seguindo uma tabela do Ábaco, assim achada muito completa, algumas páginas de texto, então a tabela de multiplicação, e várias outras tabelas, duas das quais se referem a frações: essas fraternidades parecem, a primeira vista, apresentar apenas confusão, mas reconhecemos que se destinavam a formar um tratado completo de aritmética, incluindo a exposição do sistema numeral, as regras de multiplicação e divisão, e o cálculo de frações. Neste conjunto, as tabelas tiveram que, por assim dizer, falar por si mesmas e compensar longas explicações (CHASLES, 1843a, p. 6, tradução nossa). ${ }^{4}$

Seguem as divisões explicativas quanto à operação da divisão contida no Abaco

\section{Computi:}

I - Divisão de unidades por unidades.

II - Divisão de dezenas, centenas, etc. por unidades.

III - Divisão de centenas, milhares, etc. por dezenas.

IV - Divisão de dezenas, centenas e milhares, simples ou reunidos, por unidades juntas com dezenas. Método das diferenças.

V - Divisão de outra maneira, pelo método das diferenças, de centenas, milhares, etc. por unidades juntas com dezenas. Reduzindo o dividendo a uma só unidade de sua ordem e multiplicando o quociente e o resto pela denominação do dividendo, isto é, pelo número das unidades que contém.

VI - Divisão de dezenas juntas com centenas, ou milhares, por centenas juntas com milhares, etc. Método das diferenças.

VII - Outra maneira de dividir centenas ou milhares pelos mesmos divisores, simples ou compostos. Método das diferenças.

VIII - Divisão de centenas por centenas juntas com unidades, com um lugar vazio ao meio (uno médio numerorum intermisso), ou milhar por milhar junto com dezenas (uno médio intermisso). Procedimento atual.

IX - Divisão de milhar por centenas juntas com unidades, ou dezenas de milhar por milhar juntas com dezenas. Dividindo somente uma unidade de ordem do dividendo e multiplicando o quociente e o resto pela denominação desse dividendo.

$\mathrm{X}$ - Quantas vezes um dividendo contém um divisor.

(FERREIRA, 2008, p. 54 Apud CHASLES 1843b, p. 284).

Segundo Ferreira (2008) essas regras de divisão compõe o início do texto do Abaco Computi. No entanto, na versão de Olleris (1867) que estamos analisando o

${ }^{4}$ [...] d'un tableau de l'Abacus ainsi figure quelques pages de texte, Puis la table de multipli- cation, et divers autrestableaux, dont deux relatifs aux fractions: ces fragments semblent, au premier abord, ne présenter que confusion mais on reconnait qu'ils ont été destinés former un Traité complet d'arithmétique, nant l'exposition du systeme de numération, les regles de la multiplication et de la division, et le calcul des fractions. Dans cet ensemble, les tableaux devaient, en quelque sorte, parler d'eux-mêmes et suppléer à de longues explications (CHASLES, 1843, p. 6). 
documento tem início com a explicação da multiplicação de unidades por dezenas, de dezenas por centenas, centenas por milhares e assim sucessivamente, utilizando inicialmente os algarismos romanos, comuns na época.

Percebe-se que existe uma organização lógica na divisão da obra proposta por Olleris (1867) com o interesse de favorecer o ensino e aprendizagem dos alunos acompanhados por Gerbert, pois os processos de multiplicação são apresentados de maneira descritiva.

Para além de um algoritmo que se aplique a todo e qualquer problema, as situações operatórias particulares recebem destaque, desmitificando o reducionismo ao ato de memorização algorítmica, podendo levar os professores e estudantes a compreenderem os processos históricos que conduziram aos métodos operatórios utilizados nos dias atuais.

\section{Considerações finais}

A contextualização histórica realizada neste trabalho possibilitou conhecer o sentido existencial do Abaco Computi, seu fim instrucional e educacional, ampliando o olhar da pesquisa para além do documento no passado. Vale ressaltar que não se trata de esses métodos serem aplicados para o ensino atual de maneira arbitrária. No entanto, se forem analisados na perspectiva histórica e didática poderão ser adaptados ao ensino sem descaracterizar o percurso histórico de elaboração desses conhecimentos matemáticos.

Dessa forma, levantando questionamentos para futuros trabalhos, este é um estudo em andamento cujo foco é desenvolver a interface entre a História da Matemática e o ensino, tendo como meio o uso do documento histórico Abaco Computi. Assim, poderão ser elaboradas ações que gerem reflexões sobre o percurso histórico de construção do conhecimento matemático, sendo necessário elaborar atividade que articulem a história do documento e o ensino de matemática.

\section{Referências}

BARONI, Rosa L. S.; TEIXEIRA, M. V.; NOBRE, S. R. A investigação científica em História da Matemática e suas relações com o programa de pós-graduação em Educação Matemática. In: BICUDO, M. A. V.; BORBA, M. C. Educação Matemática: pesquisa em movimento. São Paulo: Cortez, 2004. 
BROWN, D. A. The abacus and the cross: the history of the Pope who Brought the light of science to the Dark Ages. New York: Basic Books, 2010.

CHASLES, M. Histoire de l'Arithmétique: dévelloppement et détails sur divers du système de l'Abacus. Paris: 1' Academie des Sciences, 1843.

Histoire de l'Arithmétique: Explication des traités de l' Abacus, et particulièrement du traité de Gerbert. Paris: 1' Academie des Sciences, 1843.

ESTEVE, M. R. M. et al. Understanding Mathematics using original sources. Criteria and Conditions. In: BARBIN, Evelyne; KRONFELLNER, Manfred, TZANAKIS, Constantinos. (Eds.). History and Epistemology in Mathematics Education.

Proceedings of the Sixth European Summer University. Vienna: Verlag Holzhausem $\mathrm{GmbH}, 2011$, p. $415-428$.

FERREIRA, E. S. O ábaco de Silvester II. RBHM. Revista Brasileira de História da Matemática, São Paulo, v. 8, 2008.

GARNICA, A. V. M.; SOUZA, L. A. Elementos de História e de Educação Matemática. São Paulo: Cultura Acadêmica, 2012.

HAVET, J. Lettres de Gerbert (983-997). Paris: A. Picard, 1889.

LAKATOS, E. M.; MARCONI, M. de A. Metodologia do trabalho científico. 5. Ed. São Paulo: Atlas, 2003.

LOUPOT, A. Gerbert, archevêque de Reims, pape sous le nom de Sylvestre II, as vie et ses écrits. Paris: Imprimeur Editeur, 1869.

OLLERIS, A. Oeuvers de Gerbert Pape sous le nom de Sylvestre II: collationnées sur les manuscrits. Paris: LIBR.-ÉDITEU, 1867.

PEREIRA, A. C. C.; PEREIRA, D. E. Ensaio sobre o uso de fontes históricas no ensino de matemática. Rematec: Revista de Matemática, Ensino e Cultura, Natal, v. 10, n. 18, p.65-78, jan/abr. de 2015.

VASCONCELOS, F. A. História das Matemáticas na Antiguidade. Livrarias Aillaud e Bertrand: Lisboa, 1910. 